Editorial

\title{
Acknowledgement to Reviewers of Processes in 2017
}

\section{Processes Editorial Office}

MDPI AG, St. Alban-Anlage 66, 4052 Basel, Switzerland

Published: 10 January 2018

Peer review is an essential part of the publication process, ensuring that Processes maintains high quality standards for its published papers. In 2017, a total of 81 papers were published in the journal. Thanks to the cooperation of our reviewers, the median time to first decision was 20 days and the median time to publication was 48 days. The editors would like to express their sincere gratitude to the following reviewers for their time and dedication in 2017:

\begin{tabular}{|c|c|}
\hline Abatzoglou, Nicolas & Radulovic, Jovana \\
\hline Achilias, Dimitris S. & Ebrahimi, Ali \\
\hline Ahmed Isse, Abdirisak & Eda, Shigetoshi \\
\hline Ahn, Tae Seok & Diego, Luna Martínez \\
\hline Albergante, Luca & Dilonardo, Elena \\
\hline Alexis, Frank & Djabourov, Madeleine \\
\hline Álvarez, María S. & Du, Yuncheng \\
\hline Ananthasubramaniam, Bharath & Curtis, Wayne \\
\hline Andreoli, Enrico & Da Costa, Patrick \\
\hline Angiolella, Letizia & Dadlani, Aresh \\
\hline Anthony, Ben & De Martino, Andrea \\
\hline Arie, Martinus & Dejam, Morteza \\
\hline Armbruster, Udo & Derry, Matthew J. \\
\hline Avignone-Rossa, Claudio & Dewasme, Laurent \\
\hline Banga, Julio & D'hooge, Dagmar \\
\hline Barba, Francisco & Cozzolino, Daniel \\
\hline Bartocci, Pietro & Cuccato, Danilo \\
\hline Bednarz, Szczepan & Cunningham, Michael \\
\hline Bella, Federico & Cunningham, Glenn \\
\hline Bellasio, Chandra & Jyothi, Rajesh Kumar \\
\hline Beltrán, Sergio & Kameneva, Tania \\
\hline Bengtsson-Palme, Johan & Kauko, Leiviskä \\
\hline Bernstein, Elliot R. & Kim, Jong-Min \\
\hline Beuermann, Sabine & Kimura, Tomoki \\
\hline Bogel-Łukasik, Rafał & Kiparissides, Costas \\
\hline Bonalumi, Davide & Klein, Lisa C. \\
\hline Bortz, Michael & Klinke, David \\
\hline Bouet, Guenaelle & Koetz, Joachim \\
\hline Braatz, Richard & Köhler, Karsten \\
\hline Brillard, Alain & Kosinov, Nikolay \\
\hline Cadierno, Victorio & Kubo, Masataka \\
\hline Cai, Jie & Kuckling, Dirk \\
\hline Campbell, Douglas & Kumar, Gautam \\
\hline Cao, Yankai & Kunert, Renate \\
\hline Capodici, Marco & Larobina, Domenico \\
\hline Casati, Emiliano Ivan Maria & Lee, $\mathrm{Tu}$ \\
\hline Cavicchi, Kevin & Lee, Jay \\
\hline Cetin, Kristen S. & Lefebvre, Dimitri \\
\hline Chang, Hsuan & Leiza, Jose R. \\
\hline Chen, Bor-Sen & Lekakh, Simon \\
\hline
\end{tabular}


Choi, Kyu Yong

Codetta-Raiteri, Daniele

Ein-Mozaffari, Farhad

Emery, Ashley

Epelde Bejerano, Eva

Faulwasser, Timm

Feist, Adam M.

Fellows, Chris

Fisher, Lee

Font, Josep

Fontanals, Núria

Ford Versypt, Ashlee

Franchini, Giuseppe

$\mathrm{Fu}$, Qiang

Fujita, Shin-ichiro

Furlani, Maurizio

Gadouleau, Maximilien

Gao, Zhe

Gao, Hanyu

García, Míriam R.

Gentile, Piergiorgio

Georgakis, Christos

Gerogiorgis, Dimitrios I.

Gil, Antonio

Giuffrida, Antonio

Görlich, Dennis

Greco, Antonio

Grinstein, Georges

Gros, Sebastien

Grossi, Giancarlo

Hahn, Juergen

Hannemann-Tamas, Ralf

Hardiman, Gary

Hauser, Hansjoerg

Hebb, Adam O.

Hellweger, Ferdiinand

Helmer, Harald Ernst

Herrmann, Frank

Hervás-Pérez, J.P.

Hondo, Hironori

Huang, Samuel

Huang, Xingfeng

Huang, Zuyi

Huang, Hsiang-Hsi

Hutchinson, Robin A.

Meng, Qing-jun

Meyer-Kohlstock, Daniel

Mhaskar, Prashant

Miccio, Francesco

Mija, Alice

Mingareev, Ilya

Moad, Graeme

Moehlis, Jeff

Mohan, Ganesh Babu Malli

Moran, Federico

Moratti, Steve

Moxon, K. A.

Mukherjee, Rajib
Lijffijt, Jefrey

Lin, Yen-Hui

Lind, Anna

Liu, Xue

Liu, Yang

Liu, Xinfeng

Livingston, George

López-Sabirón, Ana María

Luong, Dzung Dinh

Madhuranthakam, Chandra

Malgorzata, Wisniewska

Maravelias, Christos

Markides, Christos

Martins, Ian James

Masoumi, Samira

Massobrio, Paolo

Mateus, Dina M. R.

Scholten, Kee

Scott, Alison

Seabra Dos Reis, Marco

Severson, Kristen Ann

Shaha, Sugrib Kumar

Shoemaker, Jason

Singh, Ravendra

Singh, Rajveer

Singhal, Naresh

Sorensen, Chris

Stahl, Frank

Storti, Giuseppe

Strati, Irini F.

Sung, Jaeyong

Sy, Ibrahima

Szekely, Gyorgy

Taboada, Fernando Gonzalez

Taylor, Stephanie R.

Telen, Dries

Tondi, Gianluca

Tortosa, Leandro

Transtrum, Mark K.

Tseng, Boo Shan

Tsukahara, Takahiro

Turki, Sadok

Iyer, Aparna

Jackson, Mark

Jäschke, Johannes

Jiang, Benben

Jung, Sung Kwon

Upton, Chris

Vadigepalli, Rajanikanth

Vassanelli, Stefano

Vicencio, Alfredo Núñez

Vilanova, Ramon

Vilas, Jose Luis

Visaveliya, Nikunjkumar

Wei, Xiupeng

Werker, Alan G

Whidborne, James

Wilson, Ian 
Murrugarra, David

Mustafa, Mustafa A.

Nadais, Helena

Nadal Soriano, Enrique

Neto, Diogo M.

Nicoletta, Fiore Pasquale

Nikolaou, Michael

Noordergraaf, I.W.

Nouvel, Cécile

Nurumbetov, Gabit

Okajima, Junnosuke

Oliveira, José António Vasconcelos

Omasa, Takeshi

Ozturk, Mustafa Cagdas

Palazoglu, Ahmet

Panicker, Rajesh

Pannocchia, Gabriele

Papaefthymiou, Spyros

Park, You-Jin

Pasman, Hans J.

Passaglia, Elisa

Pavlopoulos, Georgios A.

Peampermpool, Rakpong

Perc, Matjaz

Peres, Sabine

Perez-Gracia, Maria-Teresa

Pino, Lidia

Polizzi, Karen

Puglia, Debora
Wuest, Thorsten

Turkoglu, Kamran

Rahimi, Farid

Rahman, Ziaur

Rahmanian, Nejat

Rangaiah, G.P.

Raue, Andreas

Ravaghi-Ardebili, Zohreh

Renna, Paolo

Rhinehart, R. Russell

Riahinezhad, Marzieh

Risi, Michele

Robson, Barbara

Rosic, Nedeljka

Sacco, Riccardo

Safdarnejad, Seyed Mostafa

Save, Maud

Xie, Qian

$\mathrm{Xu}$, Shu

$\mathrm{Xu}$, Jiangtao

Yahyaei, Mohsen

Yao, Yuan

Yavitt, Joseph

Yazdan Panah, Nima

Yin, Shi

Zbeeb, Khaled

Zhang, Jie

Zhao, Jingzhou

(C) 2018 by the authors. Licensee MDPI, Basel, Switzerland. This article is an open access article distributed under the terms and conditions of the Creative Commons Attribution (CC BY) license (http://creativecommons.org/licenses/by/4.0/). 